\title{
Crystal structure of trimethylsilyl-triphenylphosphane-iminium- dichloroiodate $(\mathrm{I}),\left(\mathrm{C}_{6} \mathrm{H}_{5}\right)_{3}$ PNHSi $\left(\mathrm{CH}_{3}\right)_{3} \mathrm{ICl}_{2}$
}

\author{
J. Grebe, F. Weller and K. Dehnicke
}

Universität Marburg. Fachbereich Chemie. Hans Meerwein-Straße. D-35043 Marburg. Germany

Received May 28. 1996. CSD-No. 402495

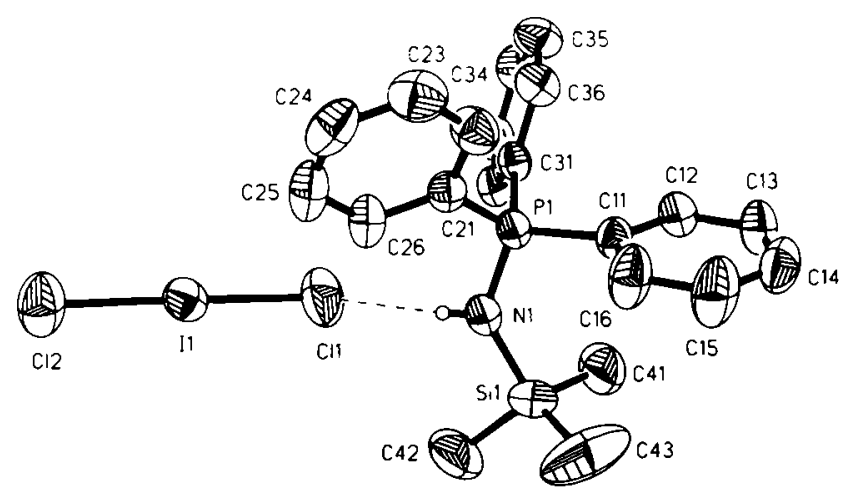

Source of material: The compound is produced by reaction of $\mathrm{Me}_{3} \mathrm{SiNPPh}_{3}$ with $\mathrm{ICl}$ in the presence $\mathrm{HCl}$ in dichloro methane solution and subsequent addition of $n$-hexane at $293 \mathrm{~K}$.

Average bond lengths in the two symmetry independent cations are: $d(\mathrm{P}-\mathrm{N})=162.2(4) \mathrm{pm} ; d(\mathrm{Si}-\mathrm{N})=176.5(4) \mathrm{pm} ; d(\mathrm{~N}-\mathrm{H})=$ $72(5) \mathrm{pm} ; d(\mathrm{H}(1) \cdots \mathrm{Cl}(1)=267(5) \mathrm{pm}$. Cation bond angles are (in average): $(\mathrm{Si}-\mathrm{N}-\mathrm{P})=132.6(3)^{\circ} ;(\mathrm{N}-\mathrm{H} \cdots \mathrm{Cl})=160(5)^{\circ}$. The anions lie in general positions with $d(\mathrm{Il}-\mathrm{Cl})=251.5(2) \mathrm{pm}$ and $256.7(2) \mathrm{pm}$ and are disordered around centres of symmetry with $d(\mathrm{I}(2)-\mathrm{Cl})=$ 252(1) $\mathrm{pm}$ and $256(1) \mathrm{pm}$ and $d(\mathrm{I}(3)-\mathrm{Cl})=247(1) \mathrm{pm}$ and 252.2(4) pm. The bond angle of anion $1(\mathrm{Cl}(1)-\mathrm{I}(1)-\mathrm{Cl}(2))$ is $178.17(5)^{\circ}$, whereas the other species are linear. Details of the bonding in the $\mathrm{ICl}_{2}^{-}$-ions are comparable with other compounds with the dichloroiodate(I)-ion (see ref. 1).

The observed bond angle SiNP is somewhat different from that in $\left[\mathrm{Me}_{3} \mathrm{SiN}(\mathrm{H}) \mathrm{PMe}_{3}\right]_{2}\left[\mathrm{Se}_{2} \mathrm{Cl}_{6}\right]\left(150.6(6)^{\circ}\right.$, see ref. 2$)$.

$\mathrm{C}_{21} \mathrm{H}_{25} \mathrm{Cl}_{2} \mathrm{INPSi}$, triclinic, $P \overline{1}$ (No. 2), $a=10.836(1) \AA$,

$b=14.035(1) \AA, c=16.324(1) \AA, \alpha=88.062(4)^{\circ}, \beta=84.880(5)^{\circ}$, $\gamma=79.806(5)^{\circ}, V=2433.2 \AA^{3}, Z=4, R(F)=0.034, R_{W}\left(F^{2}\right)=0.072$.

Table 1. Parameters used for the $\mathrm{X}$-ray data collection

\begin{tabular}{ll}
\hline Crystal: & orange prism, size $0.12 \times 0.17 \times 0.38 \mathrm{~mm}$ \\
Wavelength: & Mo $K_{\alpha}$ radiation $(0.71073 \AA)$ \\
$\mu:$ & $16.57 \mathrm{~cm}^{-1}$ \\
Diffractometer: & Enraf Nonius CAD4 \\
Scan mode: & $\omega$ \\
$T_{\text {measurement: }}$ & $293 \mathrm{~K}$ \\
$2 \theta_{\text {max }}:$ & $49^{\circ}$ \\
$\mathrm{N}\left(h k l_{\text {unique: }}\right.$ & 8240 \\
Criterion for $I_{0}:$ & $I_{0}>2 \sigma\left(I_{0}\right)$ \\
$N(\text { param })_{\text {refined }}:$ & 476 \\
Program: & SHELXL-93
\end{tabular}

Table 2. Final atomic coordinates and displacement parameters (in $\AA^{2}$ )

\begin{tabular}{|c|c|c|c|c|c|}
\hline Atom & Site & $x$ & $y$ & $z$ & $U_{\text {iso }}$ \\
\hline$H(1)$ & $2 i$ & $0.023(5)$ & $0.482(3)$ & $0.198(3)$ & $0.06(2)$ \\
\hline$H(2)$ & $2 i$ & $0.667(4)$ & $0.047(3)$ & $0.086(2)$ & $0.03(1)$ \\
\hline$H(12)$ & $2 i$ & $-0.3237(4)$ & $0.6612(3)$ & $0.3033(2)$ & $0.087(7)$ \\
\hline$H(13)$ & $2 i$ & $-0.3742(4)$ & $0.7818(3)$ & $0.4037(3)$ & $0.087(7)$ \\
\hline H(14) & $2 i$ & $-0.2554(5)$ & $0.7739(3)$ & $0.5184(2)$ & $0.087(7)$ \\
\hline$H(15)$ & $2 i$ & $-0.0861(5)$ & $0.6455(4)$ & $0.5329(2)$ & $0.087(7)$ \\
\hline$H(16)$ & $2 i$ & $-0.0357(4)$ & $0.5249(3)$ & $0.4326(3)$ & $0.087(7)$ \\
\hline $\mathbf{H}(22)$ & $2 i$ & $-0.2311(3)$ & $0.4005(3)$ & $0.4220(3)$ & $0.083(7)$ \\
\hline $\mathbf{H}(23)$ & $2 i$ & $-0.1847(4)$ & $0.2449(3)$ & $0.4824(2)$ & $0.083(7)$ \\
\hline$H(24)$ & $2 i$ & $-0.0180(5)$ & $0.1300(2)$ & $0.4236(3)$ & $0.083(7)$ \\
\hline $\mathbf{H}(25)$ & $2 i$ & $0.1021(4)$ & $0.1707(3)$ & $0.3043(3)$ & $0.083(7)$ \\
\hline$H(26)$ & $2 i$ & $0.0557(4)$ & $0.3262(3)$ & $0.2439(2)$ & $0.083(7)$ \\
\hline $\mathrm{H}(32)$ & $2 i$ & $-0.1584(3)$ & $0.5361(3)$ & $0.1100(2)$ & $0.071(6)$ \\
\hline $\mathbf{H}(33)$ & $2 i$ & $-0.3260(4)$ & $0.5425(3)$ & $0.0264(1)$ & $0.071(6)$ \\
\hline$H(34)$ & $2 i$ & $-0.5212(3)$ & $0.5087(3)$ & $0.0836(2)$ & $0.071(6)$ \\
\hline H(35) & $2 i$ & $-0.5490(3)$ & $0.4685(4)$ & $0.2244(3)$ & $0.071(6)$ \\
\hline$H(36)$ & $2 i$ & $-0.3814(4)$ & $0.4622(3)$ & $0.3081(1)$ & $0.071(6)$ \\
\hline$H(411)$ & $2 i$ & $-0.028(7)$ & $0.785(3)$ & $0.173(4)$ & $0.21(2)$ \\
\hline$H(412)$ & $2 i$ & $-0.136(4)$ & $0.734(5)$ & $0.216(3)$ & $0.21(2)$ \\
\hline$H(413)$ & $2 i$ & $-0.090(7)$ & $0.715(6)$ & $0.123(2)$ & $0.21(2)$ \\
\hline$H(421)$ & $2 i$ & $0.232(5)$ & $0.646(3)$ & $0.134(4)$ & $0.15(2)$ \\
\hline$H(422)$ & $2 i$ & $0.160(6)$ & $0.596(4)$ & $0.073(2)$ & $0.15(2)$ \\
\hline$H(423)$ & $2 i$ & $0.240(5)$ & $0.533(2)$ & $0.138(4)$ & $0.15(2)$ \\
\hline$H(431)$ & $2 i$ & $0.181(5)$ & $0.706(4)$ & $0.294(5)$ & $0.20(2)$ \\
\hline$H(432)$ & $2 i$ & $0.161(6)$ & $0.598(2)$ & $0.305(5)$ & $0.20(2)$ \\
\hline$H(433)$ & $2 i$ & $0.069(6)$ & $0.681(5)$ & $0.354(3)$ & $0.20(2)$ \\
\hline $\mathbf{H}(52)$ & $2 i$ & $0.7015(3)$ & $-0.1359(3)$ & $0.1158(2)$ & $0.075(7)$ \\
\hline H(53) & $2 i$ & $0.6018(4)$ & $-0.2724(2)$ & $0.1239(2)$ & $0.075(7)$ \\
\hline$H(54)$ & $2 i$ & $0.4328(4)$ & $-0.2806(2)$ & $0.2236(3)$ & $0.075(7)$ \\
\hline$H(55)$ & $2 i$ & $0.3635(3)$ & $-0.1523(3)$ & $0.3152(2)$ & $0.075(7)$ \\
\hline$H(56)$ & $2 i$ & $0.4633(4)$ & $-0.0158(2)$ & $0.3071(2)$ & $0.075(7)$ \\
\hline$H(62)$ & $2 i$ & $0.9343(4)$ & $0.0306(3)$ & $0.1487(2)$ & $0.088(8)$ \\
\hline$H(63)$ & $2 i$ & $1.1260(3)$ & $-0.0202(3)$ & $0.2080(3)$ & $0.088(8)$ \\
\hline$H(64)$ & $2 i$ & $1.1260(3)$ & $-0.0839(3)$ & $0.3433(3)$ & $0.088(8)$ \\
\hline$H(65)$ & $2 i$ & $0.9343(4)$ & $-0.0969(3)$ & $0.4194(2)$ & $0.088(8)$ \\
\hline$H(66)$ & $2 i$ & $0.7426(3)$ & $-0.0461(3)$ & $0.3601(2)$ & $0.088(8)$ \\
\hline $\mathrm{H}(72)$ & $2 i$ & $0.7015(3)$ & $0.1402(3)$ & $0.3528(2)$ & $0.078(7)$ \\
\hline $\mathrm{H}(73)$ & $2 i$ & $0.5752(4)$ & $0.2727(3)$ & $0.4212(2)$ & $0.078(7)$ \\
\hline $\mathrm{H}(74)$ & $2 i$ & $0.3822(4)$ & $0.3443(3)$ & $0.3717(3)$ & $0.078(7)$ \\
\hline $\mathrm{H}(75)$ & $2 i$ & $0.3153(3)$ & $0.2835(3)$ & $0.2536(3)$ & $0.078(7)$ \\
\hline$H(76)$ & $2 i$ & $0.4416(4)$ & $0.1510(3)$ & $0.1851(2)$ & $0.078(7)$ \\
\hline$H(811)$ & $2 i$ & $0.830(3)$ & $0.297(2)$ & $0.114(2)$ & $0.10(1)$ \\
\hline $\mathrm{H}(812)$ & $2 i$ & $0.854(3)$ & $0.206(3)$ & $0.173(2)$ & $0.10(1)$ \\
\hline$H(813)$ & $2 i$ & $0.722(3)$ & $0.274(3)$ & $0.177(2)$ & $0.10(1)$ \\
\hline$H(821)$ & $2 i$ & $0.611(5)$ & $0.300(2)$ & $-0.013(3)$ & $0.14(2)$ \\
\hline$H(822)$ & $2 i$ & $0.532(4)$ & $0.261(3)$ & $0.062(2)$ & $0.14(2)$ \\
\hline$H(823)$ & $2 i$ & $0.572(5)$ & $0.198(3)$ & $-0.017(2)$ & $0.14(2)$ \\
\hline$H(831)$ & $2 i$ & $0.904(5)$ & $0.189(3)$ & $-0.041(4)$ & $0.17(2)$ \\
\hline$H(832)$ & $2 i$ & $0.826(6)$ & $0.110(4)$ & $-0.060(3)$ & $0.17(2)$ \\
\hline$H(833)$ & $2 i$ & $0.930(4)$ & $0.087(3)$ & $0.002(4)$ & $0.17(2)$ \\
\hline
\end{tabular}


Table 3. Final atomic coordinates and displacement parameters (in $\AA^{2}$ )

\begin{tabular}{|c|c|c|c|c|c|c|c|c|c|c|c|}
\hline Atom & Site & Occ. & $x$ & $y$ & $z$ & $U_{11}$ & $U_{22}$ & $U_{33}$ & $U_{12}$ & $U_{13}$ & $U_{23}$ \\
\hline l(1) & $2 i$ & & $0.20551(3)$ & $0.20853(3)$ & $0.06754(2)$ & $0.0568(2)$ & $0.0770(2)$ & $0.0487(2)$ & $-0.0118(2)$ & $0.0010(1)$ & $-0.0184(2)$ \\
\hline $\mathrm{Cl}(1)$ & $2 i$ & & $0.0715(2)$ & $0.3784(1)$ & $0.06141(9)$ & $0.100(1)$ & $0.080(1)$ & $0.079(1)$ & $0.0063(9)$ & $0.0222(8)$ & $-0.0117(8)$ \\
\hline $\mathrm{Cl}(2)$ & $2 i$ & & $0.3403(2)$ & $0.0431(1)$ & $0.0692(1)$ & $0.105(1)$ & $0.082(1)$ & $0.085(1)$ & $0.0073(9)$ & $-0.0321(9)$ & $-0.0273(8)$ \\
\hline $1(2)$ & lf & & $1 / 2$ & 0 & $1 / 2$ & $0.0741(3)$ & $0.0686(3)$ & $0.0441(2)$ & $-0.0067(2)$ & $0.0067(2)$ & $-0.0122(2)$ \\
\hline $\mathrm{Cl}(3 \mathrm{~A})$ & $2 i$ & $0.51(6)$ & $0.285(1)$ & $0.014(2)$ & $0.449(1)$ & $0.066(4)$ & $0.114(8)$ & $0.080(5)$ & $0.004(4)$ & $0.003(3)$ & $-0.037(4)$ \\
\hline $\mathrm{Cl}(3 \mathrm{~B})$ & $2 i$ & $0.49(6)$ & $0.282(2)$ & $-0.022(3)$ & $0.462(1)$ & $0.091(4)$ & $0.17(1)$ & $0.066(4)$ & $-0.044(6)$ & $0.002(3)$ & $-0.031(7)$ \\
\hline $\mathrm{Cl}(4 \mathrm{~A})$ & $2 i$ & $0.32(3)$ & $0.370(3)$ & $0.591(3)$ & $0.3967(9)$ & $0.16(1)$ & $0.26(3)$ & $0.108(6)$ & $0.10(1)$ & $-0.018(7)$ & $-0.03(1)$ \\
\hline $\mathrm{Cl}(4 \mathrm{~B})$ & $2 i$ & $0.68(3)$ & $0.3966(4)$ & $0.6403(5)$ & $0.4151(4)$ & $0.083(2)$ & $0.109(4)$ & $0.073(3)$ & $-0.008(2)$ & $-0.002(2)$ & $0.009(2)$ \\
\hline $\mathbf{P}(1)$ & $2 i$ & & $0.1277(1)$ & $0.50489(8)$ & $0.71987(6)$ & $0.0376(6)$ & $0.0426(6)$ & $0.0407(6)$ & $-0.0011(5)$ & $-0.0035(5)$ & $-0.0052(5)$ \\
\hline $\mathbf{P}(2)$ & $2 i$ & & $0.6738(1)$ & $0.03606(8)$ & $0.20523(6)$ & $0.0441(6)$ & $0.0423(6)$ & $0.0355(6)$ & $-0.0088(5)$ & $-0.0012(5)$ & $-0.0093(5)$ \\
\hline $\mathrm{Si}(1)$ & $2 i$ & & $0.0598(1)$ & $0.6275(1)$ & $0.2102(1)$ & $0.0518(9)$ & $0.079(1)$ & $0.097(1)$ & $-0.0257(8)$ & $0.0031(8)$ & $-0.0104(9)$ \\
\hline $\mathrm{Si}(2)$ & $2 i$ & & $0.7375(1)$ & $0.1751(1)$ & $0.06282(8)$ & $0.0629(9)$ & $0.0532(8)$ & $0.0473(7)$ & $-0.0175(7)$ & $0.0004(6)$ & $-0.0007(6)$ \\
\hline$N(1)$ & $2 i$ & & $-0.0039(4)$ & $0.5206(3)$ & $0.2260(3)$ & $0.045(2)$ & $0.045(3)$ & $0.060(3)$ & $-0.002(2)$ & $0.004(2)$ & $-0.005(2)$ \\
\hline$C(13)$ & $2 i$ & & $-0.3048(3)$ & $0.7291(2)$ & $0.4096(2)$ & $0.068(3)$ & $0.048(3)$ & $0.069(3)$ & $0.009(3)$ & $-0.003(3)$ & $-0.012(2)$ \\
\hline$C(14)$ & $2 i$ & & $-0.2347(3)$ & $0.7245(2)$ & $0.4773(2)$ & $0.089(4)$ & $0.057(3)$ & $0.056(3)$ & $-0.013(3)$ & $0.003(3)$ & $-0.019(3)$ \\
\hline$C(15)$ & $2 i$ & & $-0.1348(3)$ & $0.6487(2)$ & $0.4859(2)$ & $0.109(5)$ & $0.086(4)$ & $0.059(3)$ & $0.005(4)$ & $-0.034(3)$ & $-0.030(3)$ \\
\hline$C(16)$ & $2 i$ & & $-0.1050(3)$ & $0.5776(2)$ & $0.4266(2)$ & $0.086(4)$ & $0.061(3)$ & $0.070(3)$ & $0.014(3)$ & $-0.034(3)$ & $-0.019(3)$ \\
\hline$C(11)$ & $2 i$ & & $-0.1751(3)$ & $0.5822(2)$ & $0.3589(2)$ & $0.044(2)$ & $0.043(3)$ & $0.039(2)$ & $-0.004(2)$ & $-0.006(2)$ & $-0.007(2)$ \\
\hline$C(22)$ & $2 i$ & & $-0.1628(2)$ & $0.3534(2)$ & $0.3979(2)$ & $0.052(3)$ & $0.059(3)$ & $0.065(3)$ & $-0.002(2)$ & $-0.004(2)$ & $0.008(3)$ \\
\hline$C(23)$ & $2 i$ & & $-0.1354(3)$ & $0.2616(2)$ & $0.4335(2)$ & $0.074(4)$ & $0.075(4)$ & $0.065(3)$ & $-0.019(3)$ & $-0.005(3)$ & $0.020(3)$ \\
\hline$C(24)$ & $2 i$ & & $-0.0371(3)$ & $0.1938(2)$ & $0.3988(2)$ & $0.093(4)$ & $0.046(3)$ & $0.079(4)$ & $-0.016(3)$ & $-0.030(3)$ & $0.005(3)$ \\
\hline$C(25)$ & $2 i$ & & $0.0339(3)$ & $0.2178(2)$ & $0.3284(2)$ & $0.083(4)$ & $0.046(3)$ & $0.078(4)$ & $0.009(3)$ & $-0.012(3)$ & $-0.015(3)$ \\
\hline$C(26)$ & $2 i$ & & $0.0065(3)$ & $0.3096(2)$ & $0.2928(2)$ & $0.066(3)$ & $0.046(3)$ & $0.055(3)$ & $0.005(2)$ & $-0.007(2)$ & $-0.008(2)$ \\
\hline$C(21)$ & $2 i$ & & $-0.0919(3)$ & $0.3774(2)$ & $0.3275(2)$ & $0.042(2)$ & $0.045(3)$ & $0.043(2)$ & $-0.006(2)$ & $-0.010(2)$ & $-0.004(2)$ \\
\hline$C(32)$ & $2 i$ & & $-0.2385(2)$ & $0.5223(2)$ & $0.1335(2)$ & $0.046(3)$ & $0.049(3)$ & $0.042(2)$ & $0.000(2)$ & $-0.002(2)$ & $-0.008(2)$ \\
\hline C(33) & $2 i$ & & $-0.3373(3)$ & $0.5260(2)$ & $0.0841(1)$ & $0.066(3)$ & $0.062(3)$ & $0.040(2)$ & $-0.002(3)$ & $-0.008(2)$ & $-0.011(2)$ \\
\hline$C(34)$ & $2 i$ & & $-0.4526(2)$ & $0.5061(2)$ & $0.1178(2)$ & $0.060(3)$ & $0.070(3)$ & $0.060(3)$ & $-0.005(3)$ & $-0.023(3)$ & $-0.010(3)$ \\
\hline$C(35)$ & $2 i$ & & $-0.4690(2)$ & $0.4824(2)$ & $0.2010(2)$ & $0.050(3)$ & $0.087(4)$ & $0.067(3)$ & $-0.017(3)$ & $-0.009(3)$ & $-0.002(3)$ \\
\hline$C(36)$ & $2 i$ & & $-0.3701(3)$ & $0.4786(2)$ & $0.2504(1)$ & $0.051(3)$ & $0.074(3)$ & $0.046(3)$ & $-0.010(3)$ & $-0.007(2)$ & $-0.002(2)$ \\
\hline$C(42)$ & $2 i$ & & $0.1864(6)$ & $0.5939(6)$ & $0.1281(6)$ & $0.074(5)$ & $0.099(5)$ & $0.192(8)$ & $-0.019(4)$ & $0.054(5)$ & $-0.001(5)$ \\
\hline$C(43)$ & $2 i$ & & $0.119(1)$ & $0.6647(8)$ & $0.3027(6)$ & $0.20(1)$ & $0.19(1)$ & $0.18(1)$ & $-0.125(9)$ & $-0.074(8)$ & $-0.024(8)$ \\
\hline $\mathrm{C}(52)$ & $2 i$ & & $0.6323(2)$ & $-0.1393(2)$ & $0.1567(2)$ & $0.055(3)$ & $0.045(3)$ & $0.048(3)$ & $-0.006(2)$ & $-0.004(2)$ & $-0.004(2)$ \\
\hline $\mathrm{C}(53)$ & $2 i$ & & $0.5734(3)$ & $-0.2198(2)$ & $0.1614(2)$ & $0.076(4)$ & $0.044(3)$ & $0.063(3)$ & $-0.005(3)$ & $-0.019(3)$ & $-0.010(2)$ \\
\hline$C(54)$ & $2 i$ & & $0.4737(3)$ & $-0.2247(2)$ & $0.2203(2)$ & $0.068(3)$ & $0.050(3)$ & $0.084(4)$ & $-0.022(3)$ & $-0.024(3)$ & $0.006(3)$ \\
\hline$C(55)$ & $2 i$ & & $0.4328(2)$ & $-0.1489(2)$ & $0.2744(2)$ & $0.062(3)$ & $0.065(3)$ & $0.069(3)$ & $-0.023(3)$ & $0.004(3)$ & $-0.001(3)$ \\
\hline$C(56)$ & $2 i$ & & $0.4917(3)$ & $-0.0684(2)$ & $0.2696(2)$ & $0.054(3)$ & $0.057(3)$ & $0.045(3)$ & $-0.012(2)$ & $-0.002(2)$ & $-0.006(2)$ \\
\hline $\mathrm{C}(51)$ & $2 i$ & & $0.5914(2)$ & $-0.0635(2)$ & $0.2107(2)$ & $0.045(2)$ & $0.042(2)$ & $0.036(2)$ & $-0.006(2)$ & $-0.005(2)$ & $-0.003(2)$ \\
\hline$C(62)$ & $2 i$ & & $0.9343(3)$ & $0.0045(2)$ & $0.2042(2)$ & $0.051(3)$ & $0.054(3)$ & $0.062(3)$ & $-0.009(2)$ & $0.003(2)$ & $-0.016(2)$ \\
\hline$C(63)$ & $2 i$ & & $1.0474(2)$ & $-0.0255(2)$ & $0.2392(2)$ & $0.044(3)$ & $0.064(3)$ & $0.091(4)$ & $-0.004(2)$ & $0.000(3)$ & $-0.022(3)$ \\
\hline$C(64)$ & $2 i$ & & $1.0474(2)$ & $-0.0631(2)$ & $0.3190(2)$ & $0.055(3)$ & $0.068(4)$ & $0.088(4)$ & $0.005(3)$ & $-0.025(3)$ & $-0.022(3)$ \\
\hline$C(65)$ & $2 i$ & & $0.9343(3)$ & $-0.0708(2)$ & $0.3639(2)$ & $0.066(4)$ & $0.083(4)$ & $0.060(3)$ & $0.004(3)$ & $-0.021(3)$ & $-0.011(3)$ \\
\hline$C(66)$ & $2 i$ & & $0.8211(2)$ & $-0.0408(2)$ & $0.3289(2)$ & $0.051(3)$ & $0.066(3)$ & $0.045(3)$ & $-0.005(2)$ & $-0.006(2)$ & $-0.003(2)$ \\
\hline $\mathrm{C}(61)$ & $2 i$ & & $0.8211(2)$ & $-0.0032(2)$ & $0.2490(2)$ & $0.044(3)$ & $0.044(3)$ & $0.046(2)$ & $-0.006(2)$ & $-0.002(2)$ & $-0.015(2)$ \\
\hline$C(72)$ & $2 i$ & & $0.6224(2)$ & $0.1695(2)$ & $0.3324(2)$ & $0.060(3)$ & $0.054(3)$ & $0.052(3)$ & $-0.009(2)$ & $-0.008(2)$ & $-0.012(2)$ \\
\hline $\mathrm{C}(73)$ & $2 i$ & & $0.5479(3)$ & $0.2477(2)$ & $0.3729(2)$ & $0.079(4)$ & $0.061(3)$ & $0.050(3)$ & $-0.012(3)$ & $0.001(3)$ & $-0.021(2)$ \\
\hline$C(74)$ & $2 i$ & & $0.4339(3)$ & $0.2900(2)$ & $0.3436(2)$ & $0.064(3)$ & $0.056(3)$ & $0.071(3)$ & $-0.006(3)$ & $0.019(3)$ & $-0.023(3)$ \\
\hline$C(75)$ & $2 i$ & & $0.3945(2)$ & $0.2541(2)$ & $0.2739(2)$ & $0.051(3)$ & $0.066(3)$ & $0.084(4)$ & $0.003(3)$ & $-0.003(3)$ & $-0.019(3)$ \\
\hline$C(76)$ & $2 i$ & & $0.4690(3)$ & $0.1759(2)$ & $0.2335(2)$ & $0.054(3)$ & $0.057(3)$ & $0.062(3)$ & $-0.003(2)$ & $-0.008(2)$ & $-0.020(2)$ \\
\hline$C(71)$ & $2 i$ & & $0.5830(2)$ & $0.1336(2)$ & $0.2628(2)$ & $0.045(3)$ & $0.042(3)$ & $0.044(2)$ & $-0.010(2)$ & $-0.001(2)$ & $-0.009(2)$ \\
\hline$C(81)$ & $2 i$ & & $0.7929(5)$ & $0.2460(4)$ & $0.1412(3)$ & $0.080(4)$ & $0.051(3)$ & $0.079(4)$ & $-0.023(3)$ & $-0.004(3)$ & $-0.007(3)$ \\
\hline $\mathrm{C}(82)$ & $2 i$ & & $0.5972(6)$ & $0.2434(5)$ & $0.0185(4)$ & $0.093(5)$ & $0.090(5)$ & $0.102(5)$ & $-0.025(4)$ & $-0.030(4)$ & $0.039(4)$ \\
\hline$C(83)$ & $2 i$ & & $0.8660(6)$ & $0.1358(5)$ & $-0.0185(4)$ & $0.107(5)$ & $0.096(5)$ & $0.073(4)$ & $-0.039(4)$ & $0.029(4)$ & $-0.006(3)$ \\
\hline
\end{tabular}

\section{References}

1. Wells, A. F.: Structural Inorganic Chemistry. Clarendon Press, Oxford 1984.

2. Folkerts, H.; Dehnicke, K.; Magull, J.; Goesmann, H.; Fenske, D.: Phosphaniminato-Trichloroselenate(II): Synthese und Kristallstrukturen von $\left[\mathrm{SeCl}\left(\mathrm{NPPh}_{3}\right)_{2}\right]^{+} \mathrm{SeCl}_{3}{ }^{-}$und $\left[\mathrm{Me}_{3} \mathrm{SiN}(\mathrm{H}) \mathrm{PMe}_{3}\right]_{2}{ }^{+}\left[\mathrm{Se}_{2} \mathrm{Cl}_{6}\right]^{2-}$. Z. Anorg. Allg. Chem. 620 (1994) 1301-1306.

3. Sheldrick, G. M.: SHELXL-93, a program for refining crystal structures. University of Göttingen, Germany 1993. 